Annuaire suisse de politique de développement

5 | 1985

Annuaire Suisse - Tiers Monde 1985

\title{
III. Politique économique extérieure
}

\section{(2) OpenEdition}

1 Journals

Édition électronique

URL : http://journals.openedition.org/aspd/1195

DOI : 10.4000/aspd. 1195

ISSN : 1663-9669

Éditeur

Institut de hautes études internationales et du développement

\section{Édition imprimée}

Date de publication : 1 janvier 1985

Pagination : 71-82

ISSN : 1660-5934

\section{Référence électronique}

«III. Politique économique extérieure », Annuaire suisse de politique de développement [En ligne], 5 |

1985, mis en ligne le 15 février 2013, consulté le 08 septembre 2020. URL : http://

journals.openedition.org/aspd/1195; DOI : https://doi.org/10.4000/aspd.1195

(c) The Graduate Institute I Geneva 


\section{POLITIQUE ECONOMIQUE EXTERIEURE}

\section{GARANTIE CONTRE LES RISQUES A L'EXPORTATION}

En 1984, le déficit de la garantie contre les risques à l'exportation (GRE) représentait $\mathbf{2 3 7}$ millions de francs, soit un montant légèrement inférieur au déficit record de l'année précédente (239 millions). Le 1er avril 1985, les taxes destinées à assurer contre les risques politiques ont été augmentées et la garantie contre les risques monétaires a été suspendue. La majeure partie des dédommagements versés par la GRE s'effectuaient pour des pertes subies lors de transferts et dans le cadre de rééchelonnement de dettes. Environ 75 pour cent des engagements de la GRE en 1984 ont concerné les exportations dans le Tiers Monde. Les oeuvres d'entraide demandent qu'on observe davantage les principes de la politique de développement lorsqu'on accorde la GRE; elles réclament aussi le droit de siéger à la Commission de la GRE ainsi qu'une meilleure transparence des opérations de la GRE.

La GRE a été créée en 1934 afin de lutter contre la crise. II s'agit d'une assurance de la Confédération contre les risques particuliers liés aux exportations. La GRE permet aux exportateurs suisses d'assurer leurs livraisons contre les risques politiques, monétaires et de fabrication (la garantie contre les risques monétaires a été suspendue le 1er avril 1985). Les garanties les plus importantes concernent cependant les risques de transferts et les consolidations de dettes (1).

La GRE a pour principe de parvenir à long terme à I'autofinancement. Les déficits persistants de ces dernières années ont nécessité des avances de la Conféderation.

En 1984, la GRE bouclait ses comptes avec des recettes de 150 millions de francs et des dépenses de 387 millions, soit une déficit de 237 millions (239 millions en 1983), qui a dû être couvert à nouveau par des avances de la Confédération; celles-ci se montent donc en tout à 599 millions de francs. En examinant les comptes de la GRE, il faut mettre en regard des avances fédérales les créances du fonds de la GRE qui proviennent des consolidations de dettes, -630 millions de francs.

Les garanties atteignaient en 1984 un montant de 5,2 milliards de francs, contre 8,4 l'année précédente. Sur ce montant, 4,8 milliards ont été mobilisés pour les garanties contre les risques politiques et 0,4 milliard contre les risques monétaires. L'engagement total de la GRE s'élève à 15,8 milliards de francs. 
Les pertes subies en cas de transferts ont connu une forte augmentation, allant jusqu'à 175 millions de francs, contre 132 millions en 1983. Les dépenses pour les consolidations de dettes se sont montées à 159 millions de francs (167 millions en 1983). II faut rappeler à ce propos que la GRE prend en charge les créances reconnues du pays débiteur. Les compensation versées pour les pertes découlant des risques monétaires ont fortement diminué, passant de 90 millions de francs en 1983 à 31 millions en 1984.

Au cours de la période considérée, la crise de l'endettement a conduit comme dans les années passées à de nombreux retards de paiements et à des négociations à long terme sur l'ajournement des dettes.

La situation difficile de la balance des paiements de nombreux pays en développement laisse présumer que la GRE devra encore faire face pendant un certain temps à des retards de paiements.

L'engagement total de la GRE qui est de 15,8 milliards de francs se répartit comme suit: 12,5 milliards de francs, soit $78,7 \%$ des garanties, sont destinées à des livraisons pour des pays en développement (conformément à la définition de l'OCDE, c'est-à-dire y compris les pays de I'OPEP et les nouveaux pays industrialisés). Une somme de 2,7 milliards $(17,2 \%)$ a été allouée aux "pays à faible revenu", dont la moitié est revenue à l'Egypte, I'Inde et I'Indonésie. En outre, 8 autres pays font l'objet d'un engagement de plus de 50 millions de francs: Chine, Salvador, Honduras, Kenya, Mali, Pakistan, Soudan, Togo.

Des paiements dans le cadre des consolidations de dettes ont été effectués sur la base de négociations avec les pays suivants: Turquie, Togo, Sénégal, Côte d'Ivoire, Sierra Leone, Soudan, Yougoslavie, Pérou, Zambie, Equateur. Dans I'ensemble, ce sont 36 accords de consolidation qui sont en vigueur avec 17 pays pour un total de 629,8 millions de francs.

L'OFAEE ne publie pas de données sur les différentes garanties de la GRE accordées selon les types de commandes ou selon les pays.

\section{Révision de l'ordonnance fédérale sur la GRE}

Le Conseil fédéral a approuvé une nouvelle révision de l'ordonnance fédérale sur la GRE. Le 1er avril 1985, les taxes relatives à la garantie contre les risques politiques ont été frappées d'une hausse de 45\%. En même temps, I'assurance contre les risques monétaires a été suspendue. Ces mesures visent à améliorer la situation financière du fonds de la GRE. Les ouvres d'entraide ont pris position au sujet de la révision de l'ordonnance fédérale sur la GRE et l'ont jugée insuffisante; quoique orientée dans la bonne direction, elle demeure unilatérale dans son essence car elle n'englobe pas la politique de développement.

\section{La GRE et la politique de développement}

Dans une pétition adressée au DFEP, la communauté de travail Swissaid/ 
Action de carême/Pain pour le prochain/Helvetas demande que la GRE $s^{\prime}$ engage sur de nouvelles voies et qu'on accorde plus d'attention aux critères de politique du développement, du point de vue de son mandat légal et de son application. Depuis 1981, la Confédération est tenue de prendre en considération les principes de politique du développement lors de ses exportations dans les pays en développement les plus paurres. Cette clause n'a pourtant jamais été invoquée pour rejeter une demande. C'est ainsi que la GRE a garanti des parts de la construction controversée du barrage de Manantali, au Mali.

Les oeuvres d'entraide critiquent également la liste des "pays en développement les plus pauvres" utilisée par la GRE et qui comprend, conformément à la classification de I'OCDE, 67 pays en développement ayant un revenu par capita inférieur à 600 dollars. La communauté de travail demande que cette catégorie inclue les pays qui ont un revenu allant jusqu'à 1400 dollars, comme cela se fait pour les mesures économiques et de politique commerciale de la coopération au développement (les crédits mixtes, par exemple).

Sur le plan pratique, les demandes doivent être examinées dans la perspective suivante: le projet envisagé va-t-il ou non renforcer l'autonomie économique du Tiers Monde?

Le matériel nucléaire, les avions pouvant servir à des fins militaires (le PC-7 par exemple) et, d'une manière générale, les produits dont "l'utilité pour l'ensemble de la population est discutable", sont à exclure de la GRE.

Finalement, les oeuvres d'entraide et les syndicats revendiquent une meilleure transparence et un droit de consultation au sein de la commission de la GRE qui est actuellement composée de représentants de l'économie et de l'administration.

Afin d'assainir la situation financière du fonds de la GRE, les oeuvres d'entraide ont fait diverses propositions, telles que les hausses de primes pour les milieux économiques. Elles demandent aussi qu'on s'abstienne d'octroyer la garantie pour de grands projets dont la valeur sur le plan de la politique du développement est douteuse, comme dans le cas de l'usine hydro-électrique d'Atatürk en Turquie. Ces organismes rejettent l'idée d'une transformation des avances fédérales en véritables subventions.

\section{Note}

1) Nous renvoyons le lecteur au compte-rendu détaillé des débats entre les différentes tendances, au sujet des questions actuelles de la GRE, qui figure dans la section "Analyses et positions" du présent annuaire.

L'étude de Markus Mugglin, "Exportrisikogarantie, Internationale Verschuldung und Schweizerische Entwicklunspolitik" (Garantie contre les risques à l'exportation, endettement international et politique suisse du développement) apporte aussi une contribution importante à ce propos; elle a été préparée à la demande de la communauté de travail et de l'Institut d'éthique sociale de la FEPS, Adliswil, 1985. 
DFEP - Communiqué de presse des 22.5.1985 et 18.3.1985

Communauté de travail Swissaid/Action de carême/Pain pour le prochain/Helvetas: Nouvelles voies pour la GRE, Lettre au DFEP du 5.2.1985

$N Z Z, 1.3 .1985$

TA, 19.3.1985

\section{AIDE MIONETAIRE INTERNATIONALE}

Dans sa session du printemps 1985 , le Parlement a prolongé de dix ans (à partir du 15.7.1985) la durée de l'arrêté fédéral qui autorise le Conseil fédéral à prendre part aux actions de soutien en faveur d'autres monnaies, afin de prévenir ou de faire cesser de graves perturbations des relations monétaires internationales. Le crédit d'engagement maximal a été réduit, passant de 2000 millions à 1000 millions de francs.

La Suisse joue un rôle important dans le cadre de l'aide monétaire internationale en raison de l'excédent de sa balance commerciale.

L'arrêté de décembre 1983 sur l'adhésion de la Suisse aux Accords généraux d'emprunt ( $A G E$ ) a eu pour effet de donner à l'aide monétaire internationale une propre base juridique. Le Conseil fédéral peut charger la Banque nationale d'effectuer les opérations nécessaires.

Le crédit maximal d'engagement a été diminué de moitié parce que la Suisse, en tant que membre des $A G E$, tient à développer ses actions principalement dans ce cadre. De plus, cette diminution du programme financier tient compte du fait que la Confédération remet à la Banque nationale le soin d'intervenir sur le front monétaire et qu'elle se limite à accorder des garanties.

Le crédit de 30 millions de dollars de la Banque nationale accordé au Portugal et garanti par la Confédération a été entièrement remboursé en septembre 1984. Ainsi, à fin 1984, il restait encore en suspens deux crédits bilatéraux garantis par la Confédération dans le cadre de l'aide monétaire internationale - pour la Turquie (45,5 millions de dollars) et la Yougoslavie (80 millions de dollars).

La communauté de travail Swissaid/Action de carême/Pain pour le Prochain/ Helvetas a demandé que l'arrêté fédéral contienne une dispositon complémentaire relative à la politique du développement; cette demande n'a pas été prise en considération. 


\section{Sources}

Arrêté fédéral sur la participation de la Suisse aux mesures monétaires internationales du 20 mars 1975.

Prise de position de la communauté de travail du 12 mars 1984.

Banque nationale, 77e Rapport de gestion, Berne 1985.

NZZ 6.9.1984 et 15.3.1985.

TA 6.9.1984.

\section{ACCORDS DE REECHELONNEMENT DES DETTES}

Les accords bilatéraux de rééchelonnement des dettes se concluent à la suite des négociations multilatérales sur le rééchelonnement qui ont lieu dans le cadre du Club de Paris.

Pendant la période étudiée, la Suisse a conclu de tels accords avec Cuba, la Yougoslavie et six pays africains en développement pour un montant total de 207 millions de francs. (Le tableau ci-après présente les détails de ces opérations.)

ACCORDS DE REECHELONNEMENT DES DETTES (1.7.1984-30.6.1985)

\begin{tabular}{lrc}
\hline & Date & Millions de francs \\
Yougoslavie & 26.7 .1984 & 60,0 \\
Côte d'Ivoire & 31.8 .1984 & 80,0 \\
Togo IV & 4.9 .1984 & 30,0 \\
Madagascar III & 14.9 .1984 & 3,7 \\
Soudan IV & 3.12 .1984 & 17,0 \\
Zambie II & 14.12 .1984 & 5,7 \\
Cuba II & 22.1 .1985 & 3,5 \\
Sénégal IV & 11.6 .1985 & 7,1 \\
Total & & $\mathbf{2 0 7 , 0}$ \\
\hline
\end{tabular}

De plus, la Suisse est en train de négocier des accords avec les pays suivants: Pérou (troisième accord), Jamaïque, Philippines, Argentine, Equateur (deuxième accord), Madagascar (quatrième accord).

Quant aux autres pays qui renégocient leurs dettes dans le cadre du Club de Paris, ils n'entrent pas en ligne de compte pour des accords bilatéraux avec 
la Suisse, étant donné que le montant minimal fixé (1 million de DTS) n'est pas atteint. II s'agit du Mozambique, du Niger, du Libéria, de la Somalie, du Costa Rica, de la Mauritanie, de la République dominicaine et du Zaîre.

Source: OFAEE

\section{PREFERENCES DOUANIERES}

En vertu de la décision relative aux préférences douanières, une grande partie des marchandises provenant des pays en développement peut entrer en Suisse en franchise. Certains produits demeurent cependant passibles de droits de douane, particulièrement ceux qui ont trait aux domaines de l'agriculture, des textiles, des vêtements et des chaussures. Dans ce cas, on applique des taux de préférences douanières et non pas les taux habituels. Dans le cadre de la septième réduction des droits de douane décidée par le GATT, le Conseil fédéral a revu à nouveau le 1er janvier 1985 les taux de préférences douanières en faveur des pays en développement.

En 1984, les importations de la Suisse en provenance des pays en développement ont représenté 6,9 milliards de francs, contre 5,9 milliards l'année précédente (1). La part des importations qui ont bénéficié des préférences douanières était de 3 milliards. Le taux d'utilisation a été de $35 \%$. Ce taux peu élevé s'explique par le fait que, pour les pierres et métaux précieux soumis à des droits très bas, on ne demande que rarement' des préférences.

La Suisse soutient financièrement l'assistance technique apportée par la CNUCED en vue d'une meilleure utilisation des préférences.

\section{Note}

1) Dans ses statistiques sur la politique économique extérieure, l'Administration fédérale compte la Yougoslavie et Israël au nombre des pays en développement, mais pas la Turquie (membre de I'OCDE).

\section{ENTRETIENS ECONOMIQUES}

Au cours de la période considérée ici, plusieurs pays en développement ont eu avec la Suisse divers entretiens à caractère économique dont nous allons donner un aperçu.

En septembre 1984, la Commission Suisse-Yougoslavie pour la coopération économique, commerciale, industrielle, scientifique et technique a discuté 
(ع) әр \% иә (9)

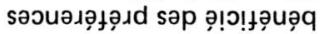

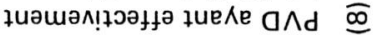

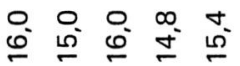

sәp әэuеuәлодd uә suo!l

-ełıodu! sap әbełuәכınod

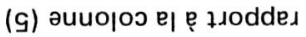
ded (9) әuиоро ए। әp $\%$ uә sәррдоээе sә6еłuene

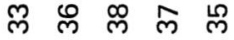

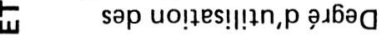

옹

ш soued әр suo!!!! uә

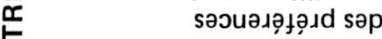

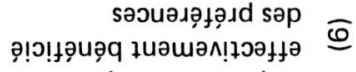

ஜ

๗் zueNe suo!jejlodu|

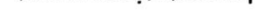

(ट) әuu잉 e| p ł Jodded ıed $(\varepsilon)$ әuиоо е әр

\% uә a^d sap әэuеuәлолd ua suo!̣ełsodu! sap lued

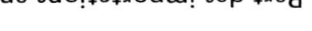

ஸ்

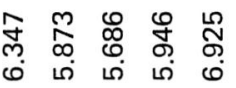

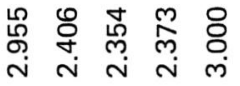

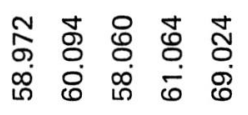

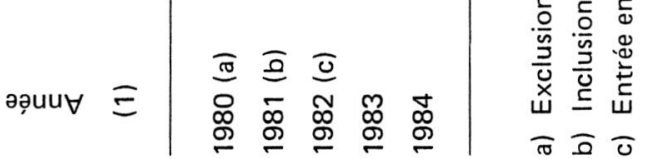


des perspectives de développement des relations commerciales bilatérales et examiné les possibiliés d'intensifier les relations mutuelles (1).

En septembre 1984, 66 exposants suisses ont pris part à la foire internationale de la construction et de l'industrie chimique à Téhéran (2).

En octobre 1984, le conseiller fédéral M. Aubert accompagné d'une délégation suisse, a abordé au cours d'un voyage en Amérique latine (Argentine, Vénézuela, Colombie, Mexique) le problème des relations économiques, en plus des question politiques. Au centre des débats figuraient la question des investissements suisses dans ces pays, I'endettement ainsi que la protection de la propriété intellectuelle pour les marques et les brevets (3).

En novembre 1984, la Suisse et l'Egypte ont signé un contrat de coopération dans le domaine de l'utilisation pacifique de l'énergie atomique. Le contrat qui doit encore être ratifié devrait permettre à l'industrie suisse d'exportation de participer au vaste programme d'implantation de centrales nucléaires en Egypte ( 6 nouvelles centrales sont prévues d'ici I'an 2005 pour une valeur de 36 milliards de dollars). Il est prévu que cette affaire fasse l'objet d'un débat au Parlement durant la session d'hiver 1985. Dans son message du 1er mai 1985, le Conseil fédéral demande au Parlement d'approuver ce contrat. Les ouvres d'entraide reprochent au message de ne pas contenir de réflexions sur la politique du développement. Avec un revenu de 580 dollars par capita (1980), I'Egypte compte parmi les pays en développement les plus pauvres; dans ce cas, conformément à la loi de la GRE, les principes de la politique suisse de développement doivent être respectés. (II faut tenir compte des demandes relatives à la GRE émanant des entreprises suisses concernées.) Selon les oeuvres d'entraide, I'option nucléaire de l'Egypte représente actuellement une erreur du point de vue de la politique du développement et c'est pourquoi la Suisse devrait "s'abstenir de contribuer à un projet de développement mal fondé à cause des mesures d'encouragement de l'Etat" (4).

La Suisse a réalisé pour la première fois une foire suisse de l'industrie (Swissexpo 84) à Djedda (Arabie saoudite) avec 130 exposants. En marge de cette exposition, le conseiller fédéral $M$. Furgler et une délégation suisse ont mené des entretiens sur l'amélioration de la coopération économique entre la Suisse et I'Arabie saoudite; on a®évoqué en particulier les questions des "joint ventures". En Jordanie, le conseiller fédéral M. Furgler a eu des entretiens portant entre autres sur le financement de projets par des crédits mixtes (5).

En janvier 1985, le symposium économique Swiss Tech 85 a eu lieu à Kuala Lumpur (Malaisie) et à Bangkok (Thaïlande). Ces manifestations ont donné l'occasion à la délégation suisse de présenter la technique et le know-how utilisés dans les exportations vers l'Asie du Sud-Est. Des séminaires sur le même thème avaient déjà été tenus en Corée du Sud, à Taïwan, en Indonésie et aux Philippines (6). 
Pour la première fois depuis que la Corée du Nord a été constituée en 1948, une délégation officielle Nord-coréenne est venue en Suisse en mars 1985 afin de participer à des entretiens économiques (7).

En mai, une délégation suisse a conduit des entretiens économiques en Corée du Sud, portant notamment sur la coopération dans les domaines des textiles et de l'agriculture. En 1984, les exportations suisses en direction de la Corée du Sud se sont élevées à 133 millions de dollars, alors que les importations Sud-coréennes représentaient 82 millions de dollars (8).

L'exposition Tecno Suiza 85, réalisée par des entreprises suisses, s'est tenue à Mexico en mai 1985. Le directeur de I'OFAEE, M. Sommaruga, a eu des entretiens avec des représentants du gouvernement sur les possibilités de développement des relations économiques bilatérales (9).

\section{Sources}

1) DFEP . Communiqué de presse, 3.9.1984.

2) NZZ, 19.9.1984.

3) NZZ, 18.10.1984.

4) NZZ, 15.11.1984 et documents de la communauté de travail Swissaid/Action de carême/Pain pour le prochain/Helvetas sur le "Contrat de collaboration Suisse-Egypte dans le domaine de l'énergie nucléaire", Berne 22.9.1985.

5) DFEP . Communiqué de presse, 19.11.1984.

6) DFEP . Communiqué de presse, 15.1.1985.

7) Le Matin, 9.3.1985.

8) DFEP . Communiqué de presse, 15.1.1985.

9) DFEP - Communiqué de presse, 9.5.1985.

\section{EXPORTATIONS D'ARMES}

Les exportations de matériel de guerre ont augmenté de 15 millions de francs en 1984 par rapport à l'année précédente, passant de 377 à 392 millions de francs. La tendance au recul de ce genre d'exportations qui s'est manifestée entre 1981 et 1983 (diminution de 134 millions de francs) n'est plus du tout sensible actuellement. Les exportations d'armes représentaient en 1984 un pourcentage toujours aussi modeste du total des exportations, soit $0,65 \%$. Les exportations à destination des pays en voie de développement (1) se sont accrues de 50 millions de francs. Le pourcentage qu'elles représentent a augmenté également, passant de $28 \%$ (en 1983) à $40 \%$ du total des exportations de matériel de guerre. A l'intérieur des pays européens en développement, ce sont les exportations d'armes vers la Grèce qui ont augmenté le plus; avec des achats d'une valeur de 67 millions de francs, la Grèce devient le principal client parmi les pays en développement. 
II faut souligner que le Nigéria ne figure plus au nombre des acheteurs de matériel de guerre suisse, alors qu'il était encore en 1983 le principal client parmi les pays en développement avec des achats de 59 millions de francs. Cette perte a été compensée par les Emirats arabes (23 millions), I'Arabie saoudite (20 millions) et Bahrein (18 millions).

Les exportations à destinations des pays en développement les plus pauvres sont restées pratiquement constantes.

Pour la première fois, en 1984, du matériel de guerre a été livré à la Chine (0,6 millions de francs - cf. tableau ci-dessous).

\section{Les avions $P C-7$}

Un débat animé s'est tenu à la session de juin 1985 du Conseil national au sujet de I'exportation d'avions $\mathrm{PC}-7$ dans des régions où règnent des conflits, tels le Guatémala et I'Iran. L'exportation d'avions Pilatus-PC-7 d'entraînement et de reconnaissance ne tombe pas sous le coup de la loi sur le matériel de guerre, selon l'arrêté fédéral du 11 mars 1985. A l'étranger, ces avions légers sont transformés en avions de combat et utilisés comme tels; cela a par exemple été révélé dans le cas du Guatémala. Un postulat Leuenberger (PS) demandait une modification de I'ordonnance relative à la loi sur le matériel de guerre; les avions dont on sait qu'ils subissent une telle transformation devraient être soumis à la loi sur le matériel de guerre. Après de longs débats, le conseil a rejeté le postulat.

L'exportation d'avions PC-7 peut être garantie paríla GRE. C'est en vain que les oeuvres d'entraide et les organisations de développement ont demandé au Conseil fédéral de renoncer à la couverture de la GRE pour les exportations de PC-7.

\section{Note}

1) La définition des pays en développement qui est utilisée ici comprend tous les pays de I'OPEP ainsi que I'Espagne, la Grèce, le Portugal, la Turquie, la Yougoslavie et Chypre (pays européens en développement). Les pays asiatiques à commerce étatisé (Chine, Mongolie, Vietnam, République populaire de Corée) ont été englobés également dans cette catégorie.

\section{Sources}

Institut d'éthique sociale de la FEPS, Berne.

NZZ 29.1. et 21.6.1985.

TA 16.3.1985. 
1983

milliers de Fr.
$\%$

milliers

de $\mathrm{Fr}$.
1984

377.236

100,0

392.332

100,0

2. Vers les pays industriels

(non compris les pays à commerce étatisé)

272.285

$72,0 \quad 234.522$

60,0

3. Vers les pays en développement * au total

104.931

28,0

157.324

40,0

Pays en développement:

sans I'OPEP

35.356

$9,5 \quad 103.629$

26,0

sans l'OPEP ni les pays en déve-

loppement européens

8.881

9,5

29.395

7,0

REPARTITION PAR GROUPES DE PAYS

Europe
Espagne
Grèce
Portugal
Turquie
autres (Yougoslavie, Chypre)
OPEP
Nigéria
Equateur
Algérie
Emirats arabes
autres (Arabie saoudite, Qatar
Koweit, Irak, Iran, Indonésie,
Vénézuela)

26.475

6.394

15.255

530

4.123

173

69.575

59.310

5.641

1.687

2.879

58

0,0

83

0,0

Autres pays en développement exportateurs de pétrole

Bahrein

0

0,0

18.733

5,0

Pays en développement à

revenu élevé

Singapour

autres (Israël, Antilles néerlan-

daises, Hongkong, Argentine)
2.032

1.976

56
1,0

1,0

1.985

1.895

1,0

1,0

90

0,0 


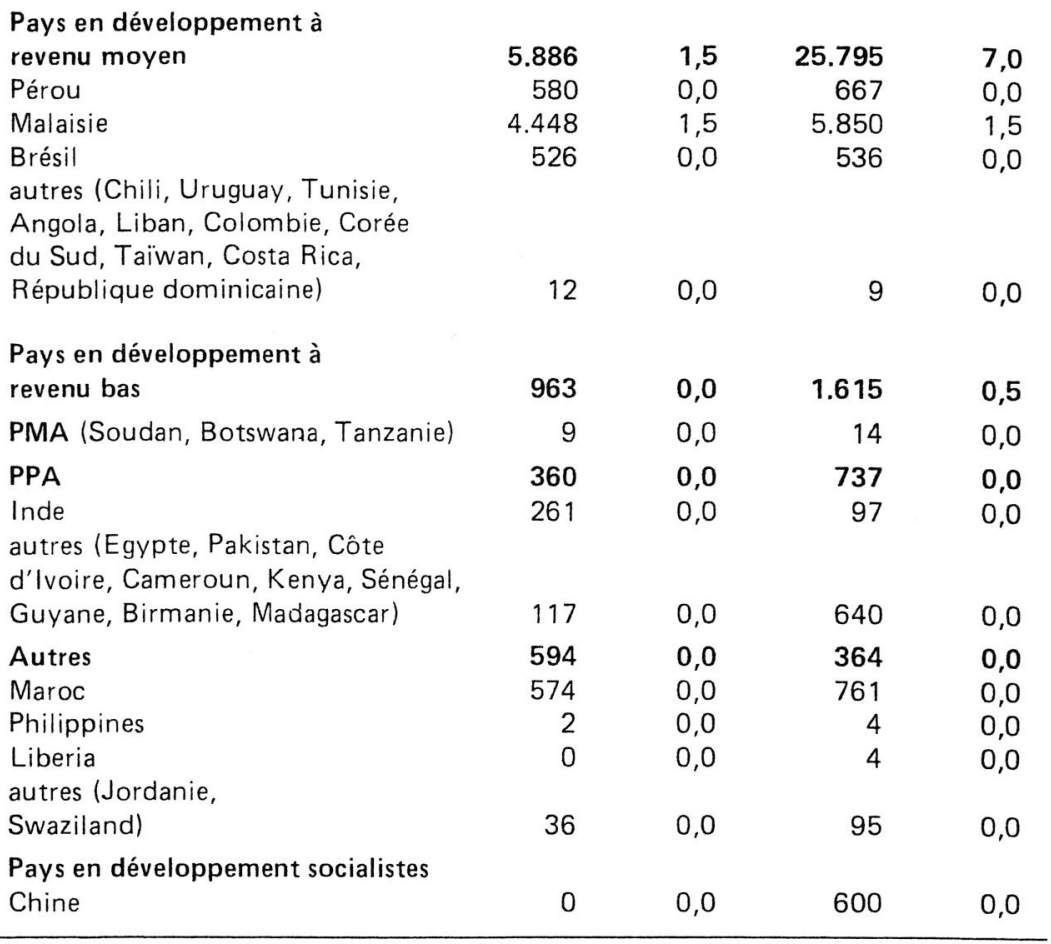

Répartition des pays en développement et adoption des critères pour la formation des groupes, selon Höhn, Der schweizerische Aussenhandel mit der Dritten Welt, Adliswil, 1980, pp. 19 ss. et annexe.

Figurent dans la liste tous les pays ayant acheté en l'espace d'un an pour Fr. 100.000 au moins, valeur à l'exportation. Les autres sont nommés, mais classés sous la dénomination "autres". 